\title{
Clinical Impact of Single and Dual Antiplatelet Therapy Beyond 12 Months on Ischemic Risk in Patients With Acute Myocardial Infarction
}

\section{OPEN ACCESS}

Edited by:

Xiaofeng Yang,

Temple University, United States

Reviewed by:

Rongxue Wu,

University of Chicago, United States

Keman Xu,

Temple University, United States

*Correspondence:

Yongcheol Kim

Yongcheo/@yuhs.ac

Specialty section:

This article was submitted to

Cardiovascular Therapeutics,

a section of the journal

Frontiers in Cardiovascular Medicine

Received: 26 September 2021 Accepted: 04 November 2021

Published: 24 November 2021

Citation:

Roh JW, Bae S, Kim Y, Son N-H, Cho D-K, Kim J-S, Kim B-K, Choi D, Hong $M-K$, Jeong $M H$, Jang $Y$ and the KAMIR-NIH Investigators (2021)

Clinical Impact of Single and Dual

Antiplatelet Therapy Beyond 12

Months on Ischemic Risk in Patients

With Acute Myocardial Infarction

Front. Cardiovasc. Med. 8:783344.

doi: 10.3389/fcvm.2021.783344

\author{
Ji Woong Roh ${ }^{1}$, SungA Bae ${ }^{1}$, Yongcheol Kim ${ }^{1 *}$, Nak-Hoon Son ${ }^{2}$, Deok-Kyu Cho ${ }^{1}$, \\ Jung-Sun Kim ${ }^{3}$, Byeong-Keuk Kim ${ }^{3}$, Donghoon Choi ${ }^{1}$, Myeong-Ki Hong ${ }^{3}$, \\ Myung Ho Jeong ${ }^{4}$, Yangsoo Jang $^{5}$ and the KAMIR-NIH Investigators
}

${ }^{1}$ Division of Cardiology, Department of Internal Medicine, Yongin Severance Hospital and Cardiovascular Center, Yonsei University College of Medicine, Yongin, South Korea, ${ }^{2}$ Division of Biostatistics, Yongin Severance Hospital, Yonsei University College of Medicine, Yongin, South Korea, ${ }^{3}$ Division of Cardiology, Severance Cardiovascular Hospital, Yonsei University College of Medicine, Seoul, South Korea, ${ }^{4}$ Division of Cardiology, Department of Internal Medicine, Chonnam National University Hospital, Chonnam University College of Medicine, Gwangju, South Korea, ${ }^{5}$ Department of Cardiology, CHA Bundang Medical Center, CHA University School of Medicine, Seongnam, South Korea

Background: There is ongoing debate regarding the optimal antiplatelet strategy beyond 12 months in patients with acute myocardial infarction (AMI) who undergo successful percutaneous coronary intervention (PCI). This study therefore aimed to investigate the clinical outcomes of single (SAPT) vs. dual antiplatelet therapy (DAPT) beyond 12 months in patients with stable AMl and second-generation drug-eluting stent (DES) implantation.

Methods: Of 13,104 patients from the Korea Acute Myocardial Infarction Registry-National Institutes of Health database, we selected 4,604 patients who underwent $\mathrm{PCl}$ with second-generation DES and exhibited no adverse clinical events within 12 months; they were classified into SAPT (aspirin or clopidogrel) or DAPT (aspirin and clopidogrel) groups. The primary endpoints were major adverse cardiac and cerebrovascular events (MACCE), including the composite of all-cause death, myocardial infarction (MI), and stroke between 12 and 36 months.

Results: The SAPT group ( $n=1,862)$ was associated with a significantly lower risk of MACCE between 12 and 36 months [4.2 vs. 8.5\%, hazard ratio (HR): 0.47, 95\% confidence interval $(\mathrm{Cl})$ : 0.37-0.61; $p<0.001$ ] than the DAPT group $(n=2,742)$. The results were consistent after adjusting for confounders through multivariable and propensity score matching analysis. Moreover, in patients with complex features (defined as an unprotected left main PCl, implanted stent length of $\geq 38 \mathrm{~mm}$, multivessel $\mathrm{PCl}$, or $\geq 3$ stents per patients), the SAPT group $(n=678)$ also demonstrated a significantly lower risk of MACCE between 12 and 36 months (4.9 vs. 9.9\%, HR: 0.46, Cl: 0.31-0.68, $p<$ 0.001) than the DAPT group $(n=1,167)$.

Conclusions: In patients with AMl who underwent successful $\mathrm{PCl}$ with second-generation DES and exhibited no adverse clinical events within 12 months, the use of SAPT was associated with a significantly lower MACCE between 12 and 36 months compared with the use of DAPT.

Keywords: acute myocardial infarction, antiplatelet therapy, aspirin, clopidogrel, major adverse cardiac and cerebral event 


\section{INTRODUCTION}

The current guidelines recommend at least 12 months of dual antiplatelet therapy (DAPT) - including aspirin with a potent $\mathrm{P}_{2} \mathrm{Y}_{12}$ inhibitor, such as ticagrelor or prasugrel-and lifelong antiplatelet therapy for patients with acute coronary syndrome (ACS) undergoing percutaneous coronary intervention (PCI) with drug-eluting stents (DESs) $(1,2)$. After 12 months of DAPT treatment, the guidelines recommend determining whether to continue DAPT, or change to single antiplatelet therapy (SAPT) by considering the risk of coronary ischemic events in individual patients (3-6). However, in recent randomized trials involving the development of DESs, evidence for short-term DAPT has increased, with favorable ischemic outcomes observed in patients with ACS who undergo second-generation DES implantation $(7,8)$. Patients with ACS remained at an increased long-term risk of ischemic events $(9,10)$; however, there are no dedicated studies regarding the optimal antiplatelet strategy beyond 12 months (SAPT vs. DAPT) in the setting of acute myocardial infarction (AMI). Moreover, it is not easy to determine the optimal strategy by performing risk stratification for each patient in daily practice; therefore, we aimed to investigate the clinical outcomes of SAPT vs. DAPT beyond 12 months in patients with AMI who underwent successful second-generation DES implantation, using a dedicated AMI registry.

\section{METHODS}

\section{Study Protocols and Population Selection}

The study population was selected from the nationwide, multicenter, prospective Korea Acute Myocardial Infarction
Registry-National Institutes of Health (KAMIR-NIH) registry. Twenty tertiary university cardiovascular centers in Korea were recruited between November 2011 and December 2015 (Supplementary Table 1); the detailed study protocols have been published previously (11). Trained research coordinators at each center collected patient data using a web-based report form on the Internet-based Clinical Research and Trial management system, supported by a grant from the Korea Centers for Disease Control and Prevention since November 2011 (iCreaT study No. C110016). The study protocols were approved by the institutional review board of each participating center and complied with the principles of the Declaration of Helsinki (IRB approval number: CNUH-2011-172). Informed consent was obtained from all patients for participation in the KAMIR registry. The standardized definitions of all variables were unified and determined by the KAMIR-NIH committee board.

The selection process of this study population is shown in Figure 1; among 13,104 patients enrolled in the KAMIR-NIH registry, we selected patients with AMI who underwent successful second-generation DES implantation without exhibiting adverse clinical events-including all-cause death, myocardial infarction (MI), stroke, revascularization, and stent thrombosis-within 12 months. The exclusion criteria included no PCI, failed PCI, or suboptimal PCI defined as the failure to restore optimal blood flow in the infarct-related coronary artery with $>50 \%$ residual stenosis and did not achieve Thrombolysis In Myocardial Infarction 2 flow after PCI; plain old balloon angioplasty alone; PCI with bare-metal stent or first-generation DES; oral anticoagulation or cilostazole use; and follow-up loss before 1 year. Additionally, we excluded patients with prolonged use of potent $\mathrm{P}_{2} \mathrm{Y}_{12}$ inhibitors, including prasugrel

\section{Korea Acute Myocardial Infarction- National Institutes of Health Registry (Nov 2011 - Dec 2015) \\ 13,104 patients with acute myocardial infarction}

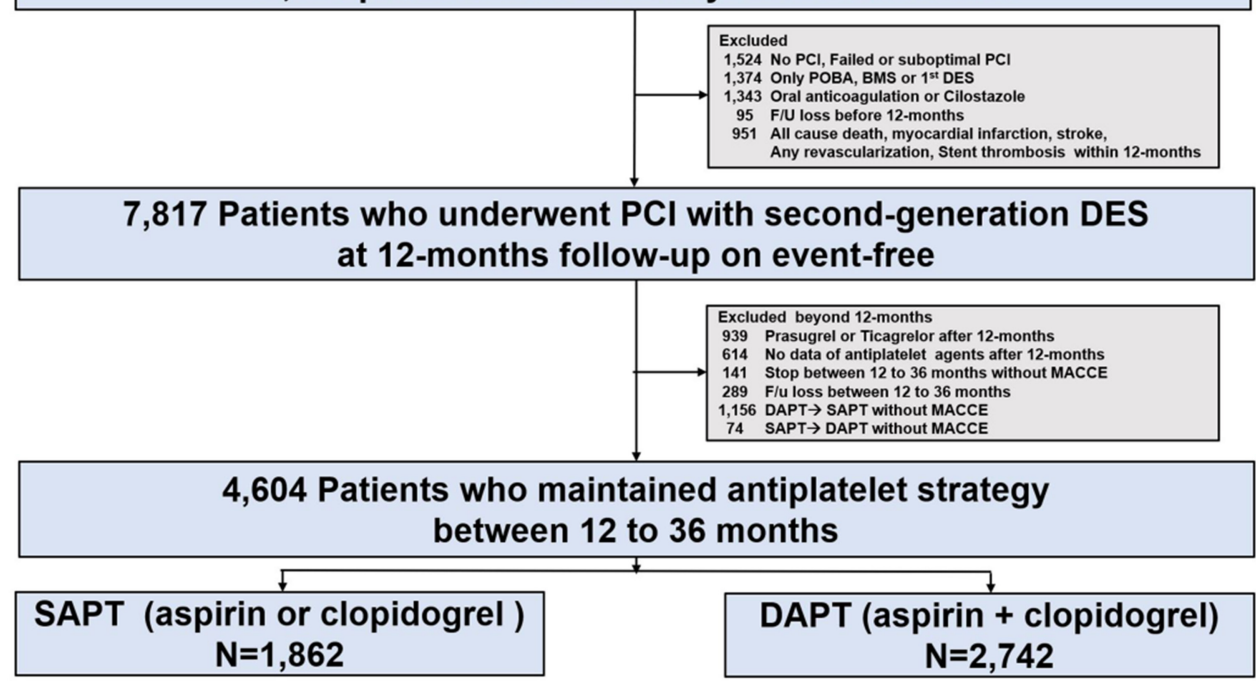

FIGURE 1 | Study flowchart. PCI, percutaneous coronary intervention; POBA, plain old balloon angioplasty; BMS, bare-metal stent; DES, drug-eluting stents; PLT, platelet; MACCE, major adverse cardiac and cerebrovascular events; SAPT, single antiplatelet therapy; DAPT, dual antiplatelet therapy. 
or ticagrelor, between 12 and 36 months; no data or terminated antiplatelet agent treatment; and who switched between DAPT and SAPT without adverse clinical events. We analyzed the clinical outcomes 12-36 months after the index procedure, stratified by antiplatelet strategy.

\section{Study Procedures}

All patients with AMI were managed according to practical guidelines $(12,13)$. Patients received loading doses of antiplatelet agents, including $300 \mathrm{mg}$ aspirin and a $\mathrm{P}_{2} \mathrm{Y}_{12}$ inhibitor (clopidogrel: $300-600 \mathrm{mg}$, ticagrelor: $180 \mathrm{mg}$, or prasugrel: $60 \mathrm{mg}$ ) before PCI. The choice of antiplatelet agent before PCIas well as the selection of treated vessels, stent type, interventional devices (e.g., intravascular modalities including intravascular ultrasound, optical coherence tomography, fractional flow reserve, and thrombosuction), and the use of glycoprotein IIb/IIIa inhibitors-was at the operator's discretion. After PCI, daily aspirin $(100 \mathrm{mg})$ and $\mathrm{P}_{2} \mathrm{Y}_{12}$ inhibitors (clopidogrel: $75 \mathrm{mg}$ once, ticagrelor: $90 \mathrm{mg}$ twice, or prasugrel: $10 \mathrm{mg}$ once daily) were prescribed.

TABLE 1 | Baseline clinical characteristics of the study population.

\begin{tabular}{|c|c|c|c|c|c|c|c|}
\hline & \multicolumn{3}{|c|}{ Crude population } & \multicolumn{3}{|c|}{ PS-matched population } & $\begin{array}{c}\text { SMD } \\
(\%)\end{array}$ \\
\hline \multicolumn{8}{|l|}{ Demographics } \\
\hline Age, years & $61.6 \pm 11.8$ & $63.2 \pm 12.2$ & $<0.001$ & $61.6 \pm 11.8$ & $61.8 \pm 12.1$ & 0.143 & 4.87 \\
\hline Male & 1454 (78.1\%) & 2108 (76.9\%) & 0.354 & $1454(78.1 \%)$ & 1437 (77.2\%) & 0.529 & 2.21 \\
\hline Body mass index & $24.1 \pm 3.1$ & $24.2 \pm 3.3$ & 0.914 & $24.1 \pm 3.1$ & $24.2 \pm 3.3$ & 0.887 & 0.48 \\
\hline Killip class III/IV & $138(7.4 \%)$ & $285(10.4 \%)$ & 0.001 & $138(7.4 \%)$ & $147(7.9 \%)$ & 0.622 & 1.85 \\
\hline STEMI & 957 (51.4\%) & 1360 (49.6\%) & 0.243 & $957(51.4 \%)$ & $951(51.1 \%)$ & 0.870 & 0.64 \\
\hline \multicolumn{8}{|l|}{ Cardiovascular risk factors } \\
\hline Hypertension & $850(45.6 \%)$ & $1349(49.2 \%)$ & 0.020 & $850(45.6 \%)$ & $865(46.9 \%)$ & 0.450 & 2.59 \\
\hline Triglyceride, mg/dL & $143.8 \pm 118.6$ & $138.1 \pm 127.9$ & 0.091 & $143.8 \pm 118.6$ & $139.6 \pm 130.2$ & 0.262 & 3.91 \\
\hline HDL cholesterol, mg/dL & $43.8 \pm 11.3$ & $43.1 \pm 11.9$ & 0.047 & $43.8 \pm 11.3$ & $43.5 \pm 11.7$ & 0.352 & 3.13 \\
\hline LDL cholesterol, mg/dL & $117.1 \pm 37.6$ & $115.7 \pm 39.2$ & 0.199 & $117.1 \pm 37.6$ & $116.0 \pm 36.0$ & 0.628 & 1.59 \\
\hline Current smoker & $824(44.3 \%)$ & $1116(40.7 \%)$ & 0.018 & $824(44.3 \%)$ & $806(43.3 \%)$ & 0.574 & 1.95 \\
\hline Family history of CVD & $128(6.9 \%)$ & $200(7.3 \%)$ & 0.258 & $128(6.9 \%)$ & $128(6.9 \%)$ & 1.000 & 0.21 \\
\hline Previous history of $\mathrm{Ml}$ & $56(3.0 \%)$ & $180(6.6 \%)$ & $<0.001$ & $56(3.0 \%)$ & $69(3.7 \%)$ & 0.275 & 4.09 \\
\hline Previous history of $\mathrm{PCl}$ & $50(2.7 \%)$ & $119(4.3 \%)$ & 0.004 & $50(2.7 \%)$ & $61(3.3 \%)$ & 0.335 & 3.65 \\
\hline Previous history of CVA & $75(4.0 \%)$ & $147(5.4 \%)$ & 0.045 & $75(4.0 \%)$ & $83(4.5 \%)$ & 0.569 & 2.19 \\
\hline LVEF (\%) & $53.1 \pm 9.7$ & $52.5 \pm 10.6$ & 0.051 & $53.1 \pm 9.7$ & $53.3 \pm 10.8$ & 0.708 & 1.35 \\
\hline Chronic kidney disease (eGFR, ml/min/1.73 $\left.\mathrm{m}^{2} \leq 60\right)$ & $606(32.5 \%)$ & $956(34.9 \%)$ & 0.110 & $606(32.5 \%)$ & $616(33.1 \%)$ & 0.753 & 1.15 \\
\hline Ticagrelor & $413(22.2 \%)$ & $551(20.1 \%)$ & & $413(22.2 \%)$ & $411(22.1 \%)$ & & \\
\hline RAS inhibitor & $1546(83.0 \%)$ & $2257(82.3 \%)$ & 0.555 & $1546(83.0 \%)$ & $1534(82.4 \%)$ & 0.634 & 1.72 \\
\hline Beta-blocker & $1654(88.8 \%)$ & 2366 (86.3\%) & 0.012 & $1654(88.8 \%)$ & 1639 (88.0\%) & 0.473 & 2.56 \\
\hline Statin & 1800 (96.7\%) & $2620(95.6 \%)$ & 0.068 & 1800 (96.7\%) & 1794 (96.3\%) & 0.655 & 1.80 \\
\hline
\end{tabular}

Values are mean $\pm S D$ or $n(\%)$.

CVA, cerebrovascular accident; CVD, cardiovascular disease; DAPT, dual antiplatelet therapy; eGFR, estimated glomerular filtration rate; HDL, high-density lipoprotein; MI, myocardial infarction; MVR, multivessel revascularization; LDL, low-density lipoprotein; LVEF, left ventricular ejection fraction; PCI, percutaneous coronary intervention; PS, propensity score; RAS, renin-angiotensin system; SAPT, single antiplatelet therapy; SMD, standard mean difference; STEMI, ST-elevation myocardial infarction. 


\section{Study Endpoints and Definitions}

The primary endpoint was major adverse cardiac and cerebrovascular events (MACCE) between 12 and 36 months after the index procedure, defined as the composite of all-cause death, MI, and stroke. All-cause death was regarded as cardiac death unless a definite, non-cardiac cause could be identified. MI was defined according to evidence of myocardial necrosis in the vascular territory of a treated vessel, i.e., the third universal definition of MI (14). Stroke was defined as the loss of neurologic function caused by an ischemic or hemorrhagic event in the brain, with symptoms lasting at least $24 \mathrm{~h}$. The secondary endpoint included individual components of MACCE and definite or probable stent thrombosis, defined according to the Academic Research Consortium definitions (15).

\section{Statistical Analysis}

Continuous variables were compared using Student's $t$-test or Mann-Whitney $U$ test, as appropriate, and are expressed as mean \pm standard deviation, or median (interquartile range). Categorical variables were compared using the Chi-square or
Fisher's exact test and are expressed as numbers and percentages. The cumulative incidence of each endpoint between 12 and 36 months was calculated based on Kaplan-Meier censoring estimates, whereas the comparison of clinical outcomes between the DAPT and SAPT groups was performed using the logrank test. To correct differences in baseline and procedural characteristics, analyses were additionally performed to adjust for confounding factors. First, a multivariable Cox regression model was used for each cutoff value, with the following covariates which was significantly affect the MACCE $(p<0.1)$ : age $\geq 65$ years, Killip class III/IV, diabetes mellitus, glucose level, history of PCI, history of cerebrovascular accident (CVA), estimated glomerular filtration rate $\leq 60 \mathrm{ml} / \mathrm{min} 1.73 \mathrm{~m}^{2}$, left ventricular ejection fraction $\leq 50 \%$, left main disease, and long stent $\geq 38 \mathrm{~mm}$. Second, we performed propensity score (PS) matching between the two groups using a multiple logistic regression model. The percent standardized mean difference after PS matching was within 10\% across all matched covariates, indicating a successful balance between the two groups. All statistical analyses were performed using the $\mathrm{R}$ statistical package

TABLE 2 | Lesion and procedural characteristics of the study population.

\begin{tabular}{|c|c|c|c|c|c|c|c|}
\hline & \multicolumn{3}{|c|}{ Crude population } & \multicolumn{3}{|c|}{ PS-matched population } & \multirow[b]{2}{*}{$\begin{array}{l}\text { SMD } \\
(\%)\end{array}$} \\
\hline & $\begin{array}{c}\text { SAPT } \\
(n=1,862)\end{array}$ & $\begin{array}{c}\text { DAPT } \\
(n=2,742)\end{array}$ & $p$ & $\begin{array}{c}\text { SAPT } \\
(n=1,862)\end{array}$ & $\begin{array}{c}\text { DAPT } \\
(n=1,862)\end{array}$ & $p$ & \\
\hline \multicolumn{8}{|l|}{ Lesion characteristics } \\
\hline Number of vessel disease & & & $<0.001$ & & & 0.592 & 1.12 \\
\hline One-vessel disease & 998 (53.6\%) & 1299 (47.4\%) & & 998 (53.6\%) & $1002(53.8 \%)$ & & \\
\hline Culprit vessel & & & 0.007 & & & 0.219 & 1.46 \\
\hline LM & $39(2.1 \%)$ & $99(3.6 \%)$ & & $39(2.1 \%)$ & $46(2.5 \%)$ & & \\
\hline LAD & 857 (46.1\%) & $1291(47.1 \%)$ & & $857(46.1 \%)$ & 865 (46.5\%) & & \\
\hline LCX & 349 (18.7\%) & $450(16.4 \%)$ & & 349 (18.7\%) & 304 (16.3\%) & & \\
\hline $\mathrm{RCA}$ & $617(33.1 \%)$ & $902(32.9 \%)$ & & $617(33.1 \%)$ & $647(34.7 \%)$ & & \\
\hline Thrombus aspiration & $549(29.5 \%)$ & $627(22.9 \%)$ & $<0.001$ & $549(29.5 \%)$ & $510(27.4 \%)$ & 0.167 & 4.59 \\
\hline Image guided $\mathrm{PCl}$ * & $487(26.2 \%)$ & $564(20.6 \%)$ & $<0.001$ & 487 (26.2\%) & $441(23.7 \%)$ & 0.081 & 6.23 \\
\hline \multicolumn{8}{|l|}{ Stent type } \\
\hline BP-BES & $412(22.1 \%)$ & $606(22.1 \%)$ & $<0.001$ & $412(22.1 \%)$ & $400(21.5 \%)$ & 0.291 & 3.24 \\
\hline DP-EES & $843(45.3 \%)$ & 1502 (54.8\%) & & $843(45.3 \%)$ & $850(45.6 \%)$ & & \\
\hline DP-ZES & 539 (28.9\%) & $552(20.1 \%)$ & & 539 (28.9\%) & 534 (28.7\%) & & \\
\hline Other (Sirolimus, Novolimus) & $68(3.7 \%)$ & $82(3.0 \%)$ & & 68 (3.7\%) & $78(4.2 \%)$ & & \\
\hline Stent diameter & $3.2 \pm 0.5$ & $3.1 \pm 0.4$ & $<0.001$ & $3.2 \pm 0.5$ & $3.1 \pm 0.9$ & 0.226 & 3.87 \\
\hline Total stent length & $28.9 \pm 13.3$ & $30.0 \pm 14.5$ & 0.010 & $28.9 \pm 13.3$ & $29.1 \pm 13.4$ & 0.648 & 1.51 \\
\hline Total stent number & $1.4 \pm 0.7$ & $1.5 \pm 0.8$ & $<0.001$ & $1.4 \pm 0.7$ & $1.4 \pm 0.8$ & 0.331 & 3.87 \\
\hline
\end{tabular}

Values are mean $\pm S D$ or $n(\%)$.

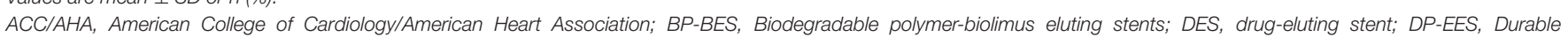

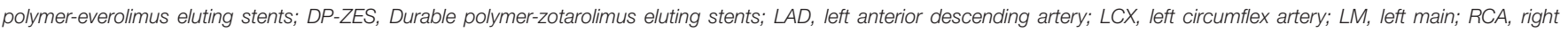
coronary artery.

Other abbreviations as in Table $\mathbf{1}$.

*Imaged guided PCl including intravascular ultrasound and optical coherence tomography. 


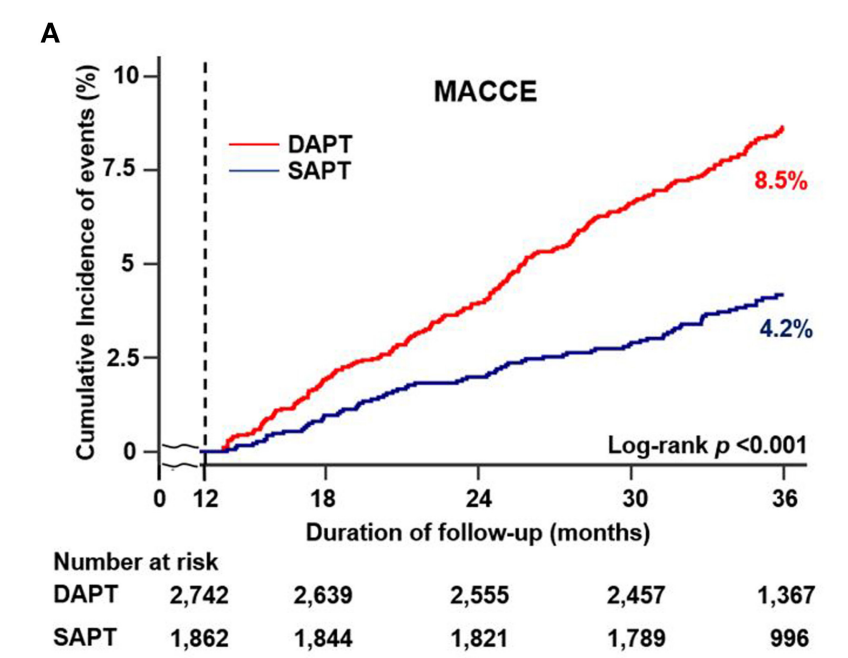

B

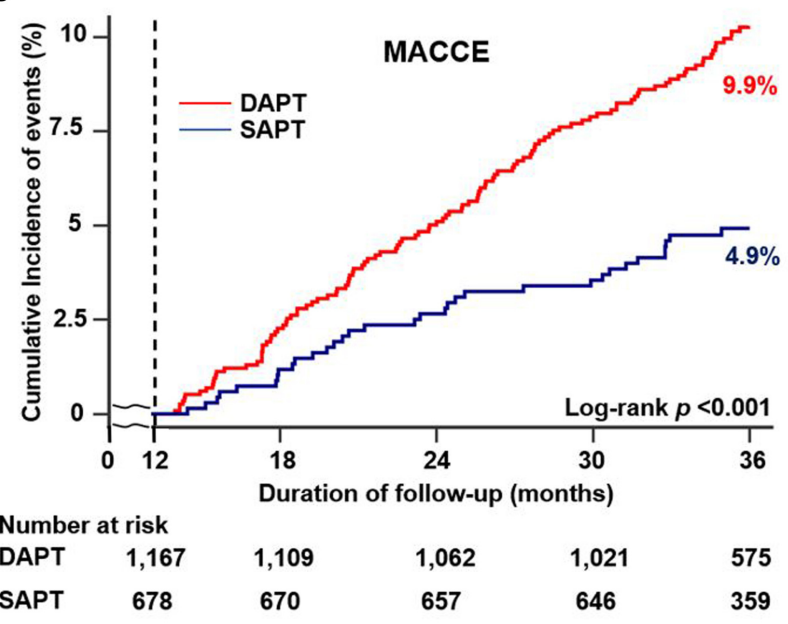

FIGURE 2 | Cumulative incidence of clinical outcomes according to antiplatelet agents between 12 and 36 months. Kaplan-Meier curves comparing the rates of MACCE between SAPT and DAPT in (A) all patients and (B) patients with complex features. MACCE, major adverse cardiac and cerebrovascular events; SAPT, single antiplatelet therapy; DAPT, dual antiplatelet therapy.

version 3.6.3 software (R Foundation for Statistical Computing, Vienna, Austria) and SPSS 25.0 for Windows (IBM Corp., Armonk, NY, USA).

\section{RESULTS}

\section{Baseline Characteristics}

Among the 4,604 patients with AMI who underwent PCI with second-generation DES and exhibited no adverse clinical events for 12 months, $1,862(40.4 \%)$ and 2,742 (59.6\%) patients were classified into the SAPT (aspirin, $n=1,347$ or clopidogrel, $n=$ 515 ) and DAPT groups, respectively. The age of the entire study population was $62.4 \pm 12.1$ years, and 3,562 patients $(77.4 \%)$ were male; 2,317 patients (50.3\%) presented with ST-elevation myocardial infarction (STEMI). The baseline clinical, lesion, and procedural characteristics of the two groups are summarized in Tables 1, 2. Patients in the SAPT group were younger, with a lower prevalence of Killip class III/IV; hypertension; diabetes mellitus; and previous history of MI, PCI, and CVA than the DAPT group. Regarding lesion and procedural characteristics, the SAPT group had a lower prevalence of multivessel disease, left main disease, ACC/AHA B2/C lesions, and glycoprotein IIb/IIIa inhibitor use; conversely, they had a higher prevalence of transradial approaches and image-guided PCI-including intravascular ultrasound or optical coherence tomography-than the DAPT group. Regarding the implanted stents, a significantly larger stent diameter, shorter total stent length, and fewer total stent numbers were observed in the SAPT group. After PS matching, the standardized differences between the groups were $<10 \%$ for all variables, indicating proper matches. No significant differences existed regarding the baseline, lesion, and procedural characteristics between the groups in the PS-matched population.

\section{Benefits of SAPT Than DAPT Beyond 12 Months for the Clinical Outcomes in Patients With AMI}

Figures 2, 3, and Table 3 compare the clinical outcomes between the groups; the follow-up duration was 1,102 (interquartile range: 1,060-1,140) days. The risk of MACCE between 12 and 36 months was significantly lower in the SAPT than in DAPT group [ 4.2 vs. $8.5 \%$, hazard ratio (HR): $0.47,95 \%$ confidence interval (CI): $0.37-0.61 ; p<0.001$; Figure 2A], primarily due to a significantly lower risk of all-cause death $(2.8$ vs. $5.1 \%$, HR: $0.54,95 \%$ CI: $0.40-0.74 ; p<0.001)$ and MI (0.7 vs. $2.2 \%$, HR: $0.31,95 \%$ CI: $0.17-0.56 ; p<0.001)$ in the SAPT group (Figures 3A,B; Table 3). Adjusted analysis using multivariable Cox regression and PS matching consistently demonstrated a significantly lower risk of MACCE, all-cause death, and MI in the SAPT group than in the DAPT group. There were no significant differences regarding the risk of noncardiac death, stroke, and stent thrombosis between the groups (Figures 3C,D; Table 3).

\section{Clinical Benefits of SAPT Than DAPT Beyond 12 Months in Patients With AMI and Complex Features}

We compared the clinical outcomes by antiplatelet strategy between 12 and 36 months in patients with AMI and complex features. Considering the clinical condition of AMI and referring to the reference, the following were considered complex features: (1) unprotected left main PCI, (2) long lesion (implanted stent length $\geq 38 \mathrm{~mm}$ ), (3) multivessel PCI, or (4) multiple stent implantation ( $\geq 3$ stents per patient) (16). Of the 1,862 patients with SAPT, 678 (36.4\%) had one or more complex feature, whereas among the 2,742 patients with DAPT, 1,167 (42.6\%) had complex features. The SAPT 


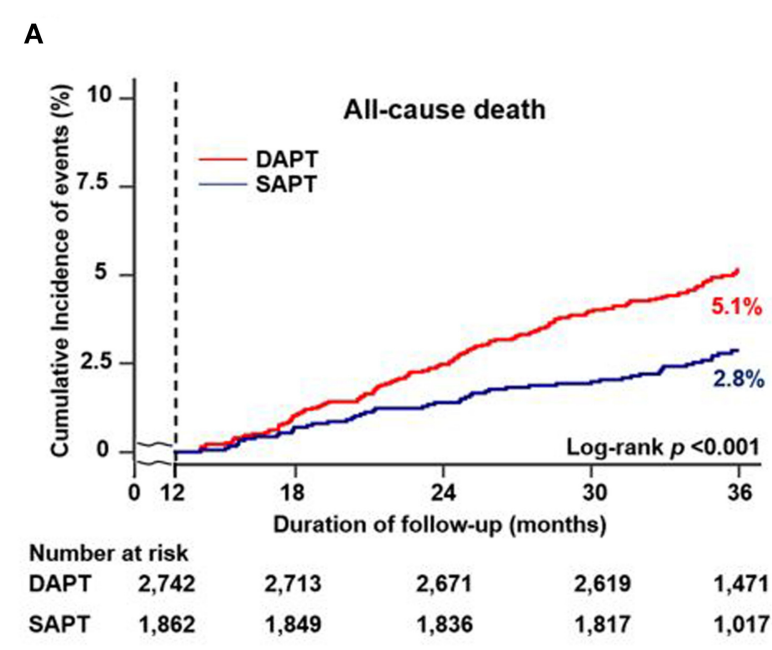

C

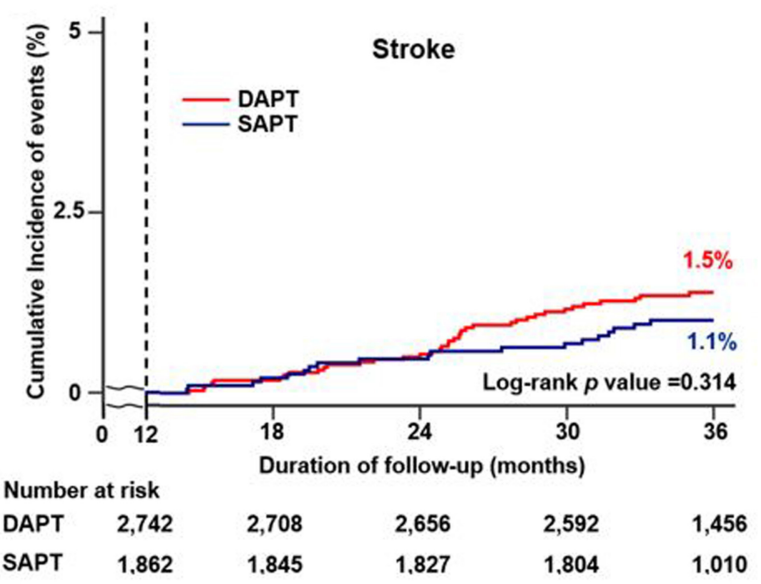

B

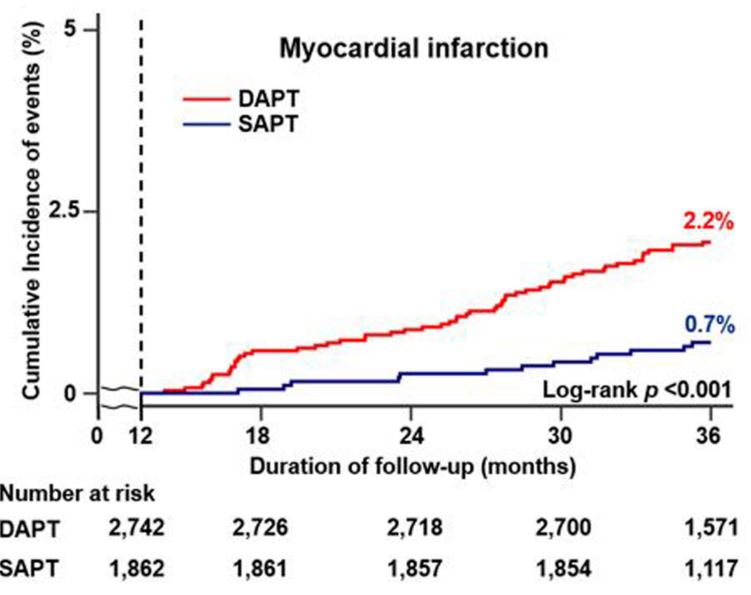

D

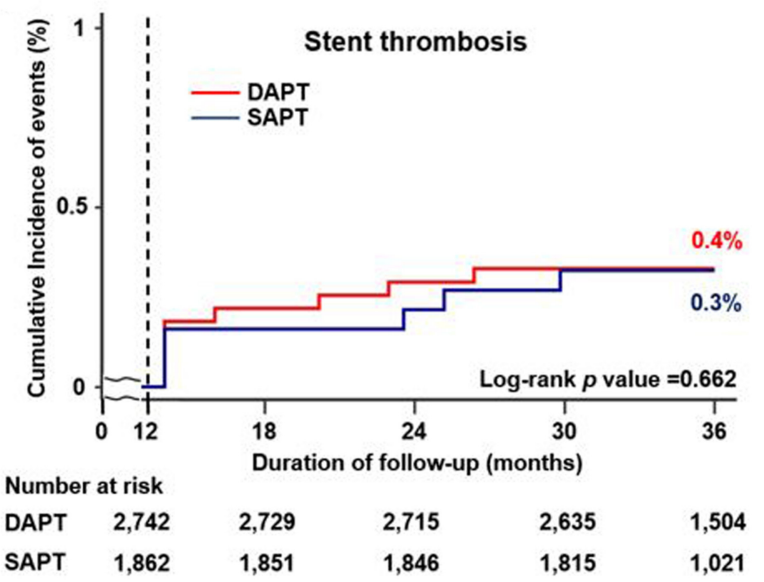

FIGURE 3 | Cumulative incidence of individual clinical outcomes according to antiplatelet agents between 12 and 36 months. Kaplan-Meier curves comparing the rates of (A) all-cause death, (B) myocardial infarction, (C) stroke, and (D) stent thrombosis between SAPT and DAPT. MACCE, major adverse cardiac and cerebrovascular events; SAPT, single antiplatelet therapy; DAPT, dual antiplatelet therapy.

group demonstrated a lower proportion of left main PCI, stent length of $\geq 38 \mathrm{~mm}$, multivessel PCI and, $\geq 3$ stent implantations compared with the DAPT group (Figure 4). Regarding the clinical outcomes, the SAPT group demonstrated a consistently lower rate of MACCE (4.9 vs. 9.9\%, HR: 0.46, 95\% CI: $0.31-$ $0.68 ; p<0.001)$ and MI (0.6 vs. $2.4 \%$, HR: $0.24,95 \% \mathrm{CI}$ : $0.08-0.68 ; p<0.007)$ between 12 and 36 months than the DAPT group in the adjusted analysis (Figure 2B; Table 4; Supplementary Figure 1B). Adjusted analysis showed that the risk of all-cause mortality, stroke, and stent thrombosis was not different between the two groups (Supplementary Figure 1; Table 4).

\section{Subgroup Analysis}

Figure 5 shows the prognostic impact of antiplatelet strategy beyond 12 months on the MACCE among the various subgroups using multivariable-adjusted hazard ratios. The lower risk of
MACCE observed in the SAPT vs. DAPT group was consistent across all subgroups; moreover, there was no significant interaction among the subgroups.

\section{DISCUSSION}

This study compared clinical outcomes between 12 and 36 months according to antiplatelet strategy in patients with AMI who underwent successful second-generation DES implantation-with no clinical events within 12 months-using data from a nationwide, multicenter, dedicated AMI registry. The main findings of the study were as follows: (1) the prevalence of MACCE beyond 12 months was significantly lower in the SAPT group (Figure 6), consistently observed with confounder adjustment by multivariable analysis and PS matching; (2) in AMI with complex features, SAPT was also associated with a significantly lower risk of MACCE between 
TABLE 3 | Comparison of clinical outcomes.

\begin{tabular}{|c|c|c|c|c|c|c|c|c|}
\hline & \multirow{2}{*}{$\begin{array}{c}\text { SAPT } \\
(n=1,862)\end{array}$} & \multirow{2}{*}{$\begin{array}{c}\text { DAPT } \\
(n=2,742)\end{array}$} & \multicolumn{2}{|c|}{ Unadjusted } & \multicolumn{2}{|c|}{ Adjusted $^{\dagger}$} & \multicolumn{2}{|c|}{ PS-adjusted } \\
\hline & & & HR (95\% Cl) & $p$-value & HR $(95 \% \mathrm{Cl})$ & $p$-value & HR (95\% Cl) & $p$-value \\
\hline MACCE $^{\star}$ & $79(4.2 \%)$ & 233 (8.5\%) & $0.47(0.37-0.61)$ & $<0.001$ & $0.54(0.42-0.70)$ & $<0.001$ & $0.54(0.41-0.71)$ & $<0.001$ \\
\hline All-cause death & $53(2.8 \%)$ & 141 (5.1\%) & $0.54(0.40-0.74)$ & $<0.001$ & $0.64(0.47-0.89)$ & 0.007 & $0.64(0.45-0.90)$ & 0.010 \\
\hline Cardiac death & $27(1.5 \%)$ & 85 (3.1\%) & $0.46(0.30-0.72)$ & 0.001 & $0.57(0.37-0.88)$ & 0.011 & $0.55(0.34-0.88)$ & 0.012 \\
\hline Noncardiac death & $26(1.3 \%)$ & $56(2.0 \%)$ & $0.66(0.42-1.06)$ & 0.084 & $0.75(0.47-1.21)$ & 0.237 & $0.78(0.47-1.30)$ & 0.340 \\
\hline $\mathrm{Ml}$ & $13(0.7 \%)$ & $61(2.2 \%)$ & $0.31(0.17-0.56)$ & $<0.001$ & $0.33(0.18-0.60)$ & $<0.001$ & 0.37 (0.19-0.69) & 0.002 \\
\hline Target vessel Ml & $3(0.2 \%)$ & $20(0.7 \%)$ & $0.22(0.06-0.73)$ & 0.013 & $0.22(0.06-0.73)$ & 0.014 & $0.30(0.08-1.07)$ & 0.064 \\
\hline Non-target vessel MI & $10(0.5 \%)$ & $41(1.5 \%)$ & $0.35(0.18-0.70)$ & 0.003 & $0.37(0.18-0.80)$ & 0.011 & 0.39 (0.19-0.82) & 0.013 \\
\hline Stroke & $21(1.1 \%)$ & $40(1.5 \%)$ & $0.76(0.45-1.29)$ & 0.315 & $0.83(0.49-1.41)$ & 0.493 & $0.77(0.44-1.36)$ & 0.369 \\
\hline Definite/probable ST & $6(0.3 \%)$ & $11(0.4 \%)$ & $0.80(0.30-2.17)$ & 0.663 & $0.87(0.32-2.37)$ & 0.777 & $0.86(0.29-2.55)$ & 0.779 \\
\hline
\end{tabular}

*MACCE: a composite of all-cause death, Ml, and stroke.

${ }^{\dagger}$ Adjusted variable: age $\geq 65$ years, Killip class $3 / 4$, DM, glucose level, history of PCl, CVA, eGFR $\leq 60 \mathrm{ml} / \mathrm{min} / 1.73 \mathrm{~m}{ }^{2}, L V E F<50 \%$, left main disease, long stent $\geq 38 \mathrm{~mm}$. Values are $n(\%)$ unless otherwise indicated.

HR, hazard ratio; Cl, confidence interval; PS, propensity score; MACCE, major adverse cardiovascular and cerebrovascular events; ST, stent thrombosis.

Other abbreviations as in Table 1.

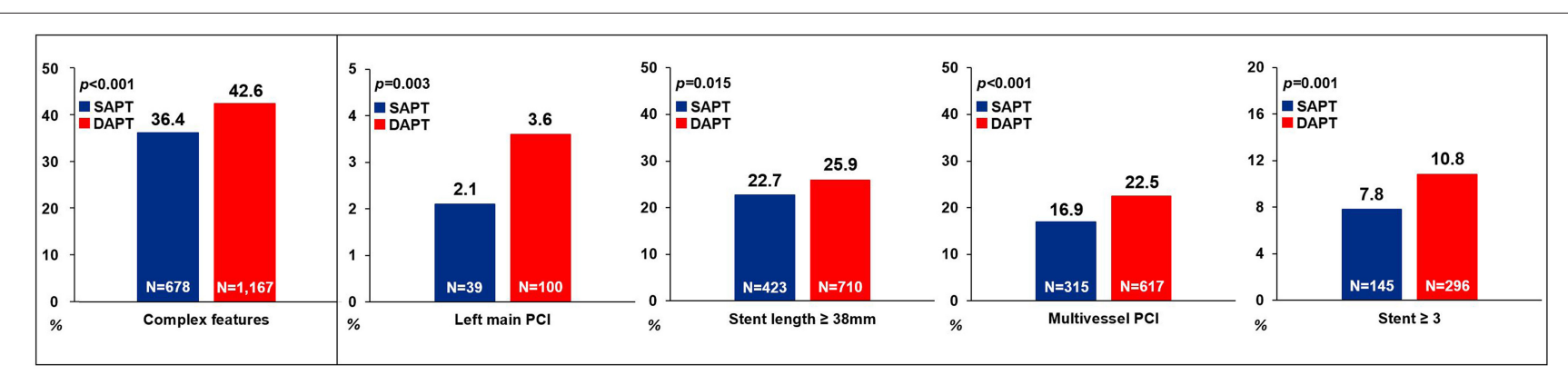

FIGURE 4 | Comparison of complex features between the SAPT and DAPT groups. SAPT, single antiplatelet therapy; DAPT, dual antiplatelet therapy; PCI, percutaneous coronary intervention.

TABLE 4 | Comparison of clinical outcomes in patients with complex features.

\begin{tabular}{|c|c|c|c|c|c|c|}
\hline & \multirow{2}{*}{$\begin{array}{c}\text { SAPT } \\
(n=678)\end{array}$} & \multirow{2}{*}{$\begin{array}{c}\text { DAPT } \\
(n=1,167)\end{array}$} & \multicolumn{2}{|c|}{ Unadjusted } & \multicolumn{2}{|c|}{ Adjusted $^{\dagger}$} \\
\hline & & & HR (95\% Cl) & $p$ & HR (95\% Cl) & $p$ \\
\hline MACCE $^{*}$ & 33 (4.9\%) & 116 (9.9\%) & $0.46(0.31-0.68)$ & $<0.001$ & $0.53(0.36-0.79)$ & 0.002 \\
\hline All-cause death & $23(3.4 \%)$ & $73(6.3 \%)$ & $0.53(0.33-0.85)$ & 0.008 & $0.66(0.41-1.07)$ & 0.088 \\
\hline Cardiac death & $11(1.6 \%)$ & 45 (3.9\%) & $0.42(0.22-0.80)$ & 0.009 & $0.53(0.27-1.04)$ & 0.066 \\
\hline Noncardiac death & $12(1.8 \%)$ & $28(2.4 \%)$ & $0.72(0.37-1.41)$ & 0.337 & $0.76(0.42-1.50)$ & 0.256 \\
\hline $\mathrm{Ml}$ & $4(0.6 \%)$ & $28(2.4 \%)$ & $0.24(0.08-0.68)$ & 0.007 & $0.30(0.10-0.85)$ & 0.024 \\
\hline Target vessel Ml & $1(0.1 \%)$ & $11(0.9 \%)$ & $0.15(0.02-1.18)$ & 0.072 & $0.19(0.05-1.30)$ & 0.124 \\
\hline Non-target vessel Ml & $3(0.4 \%)$ & $17(1.5 \%)$ & $0.30(0.09-1.01)$ & 0.051 & $0.47(0.15-1.40)$ & 0.174 \\
\hline Stroke & $11(1.6 \%)$ & 19 (1.6\%) & $0.98(0.47-2.06)$ & 0.962 & $1.03(0.48-2.18)$ & 0.945 \\
\hline Definite/probable ST & $2(0.3 \%)$ & $6(0.5 \%)$ & $0.57(0.12-2.84)$ & 0.495 & $0.47(0.09-2.44)$ & 0.370 \\
\hline
\end{tabular}

*MACCE: a composite of all-cause death, any MI, any stroke.

${ }^{\dagger}$ Adjusted variable: age $\geq 65$ years, Killip class $3 / 4, \mathrm{DM}$, glucose level, history of PCl, CVA, eGFR $\leq 60 \mathrm{~mL} / \mathrm{min} / 1.73 \mathrm{~m}{ }^{2}, \mathrm{LVEF}<50 \%$, left main disease, long stent $\geq 38 \mathrm{~mm}$. values are $n(\%)$ unless otherwise indicated.

Abbreviations as in Tables 1, 3.

12 and 36 months than DAPT; and (3) the benefits of SAPT for MACCE were consistent across diverse subgroups, including each complex feature.
Previous randomized clinical trials-including $\sim 20 \%$ of patients treated with PCI-did not show significant benefits with prolonged DAPT (aspirin and clopidogrel) in reducing 


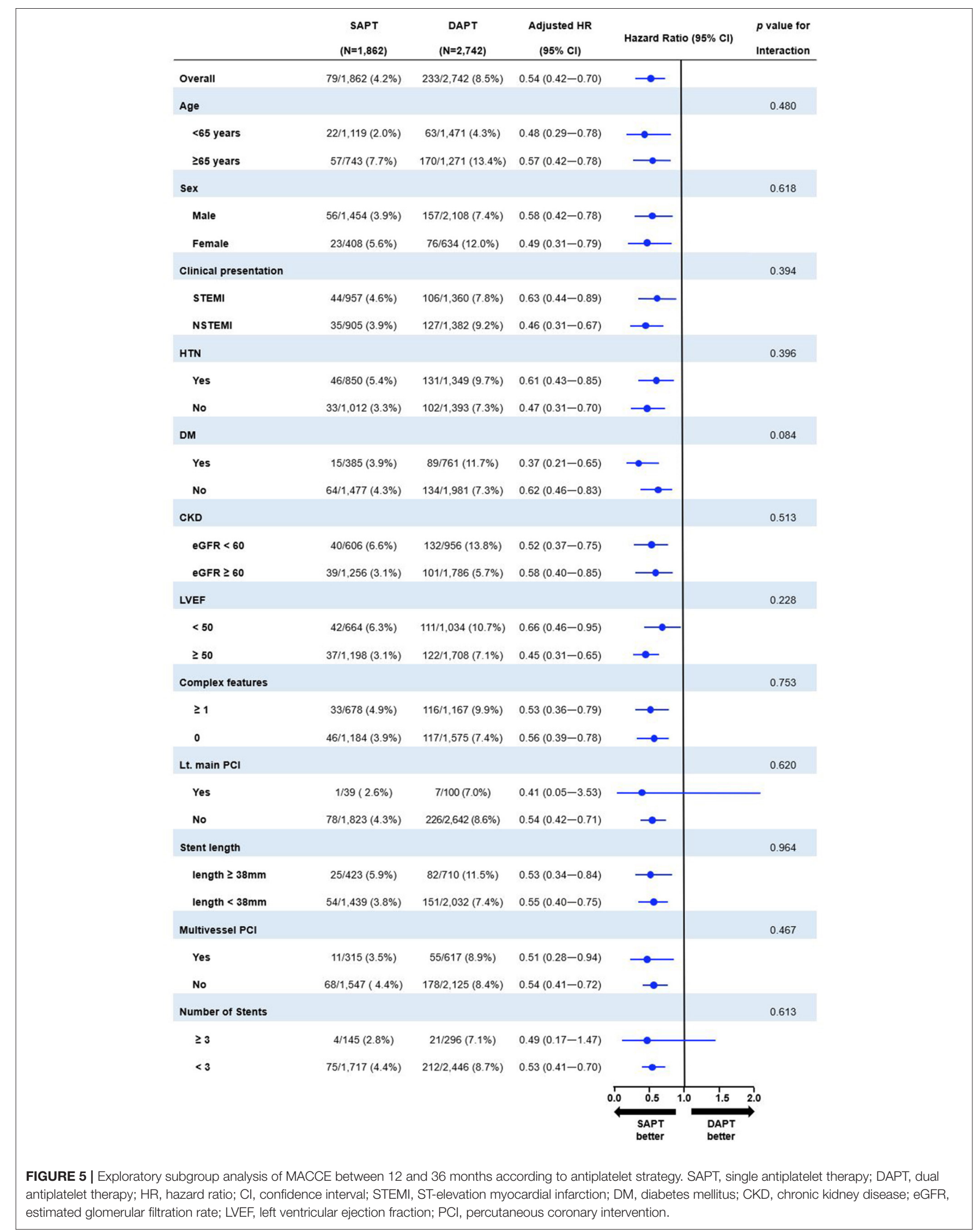




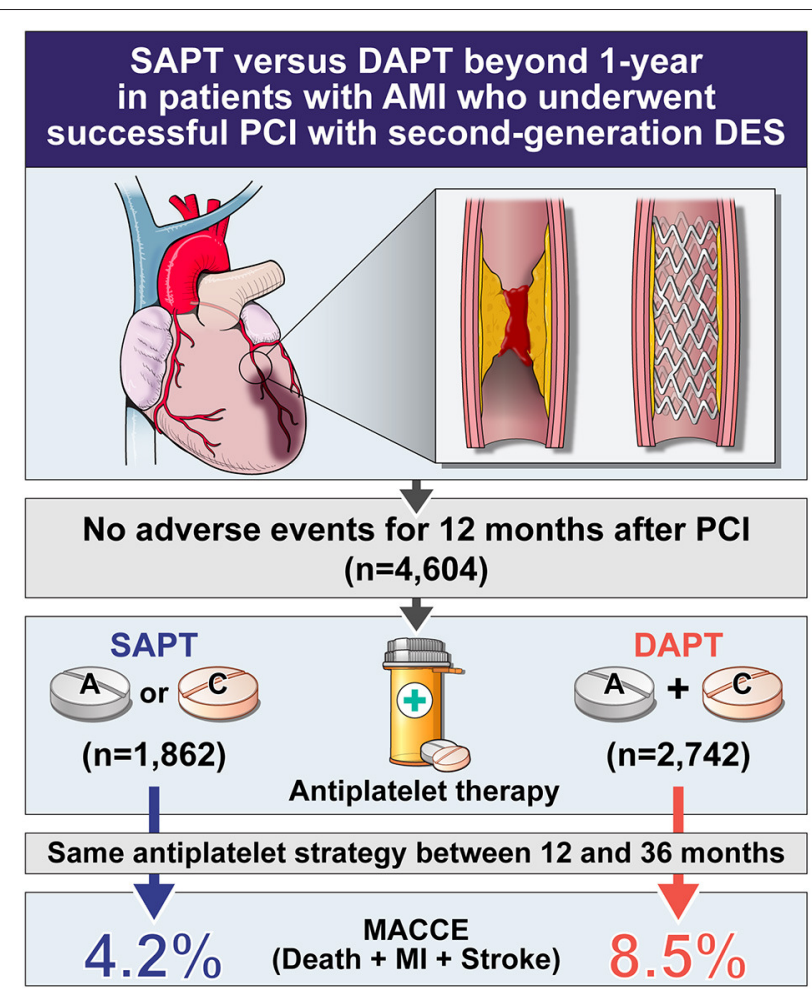

FIGURE 6 | Central illustration of this study. SAPT, single antiplatelet therapy; DAPT, dual antiplatelet therapy; AMI, acute myocardial infarction; PCl, percutaneous coronary intervention; DES, drug eluting stent; MACCE, major adverse cardiac and cerebrovascular events.

MACCE for 28 months and possibility of bleeding compared with SAPT (aspirin) (17). However, subgroup analysis in patients with prior MI, stroke, or symptomatic peripheral artery disease demonstrated that prolonged DAPT was more effective than SAPT at reducing ischemic events (18). In another randomized trial, all patients with first- or secondgeneration DES implantation and prolonged DAPT using clopidogrel or prasugrel plus aspirin exhibited a significantly lower risk of stent thrombosis and MACCE than SAPT (aspirin); however, moderate-to-severe bleeding was significantly more frequent, and the rate of all-cause death was higher in the DAPT group (19). Through these two, important, large-scale randomized clinical trials, it was ascertained that prolonged DAPT did not improve mortality, while increasing bleeding events. Furthermore, a meta-analysis of six trials including 21,457 patients with DES implantation demonstrated no benefit of prolonged DAPT in patients with stable coronary artery disease regarding overall ischemic outcomes, even MI (20).

In this study, the prolonged DAPT (aspirin and clopidogrel) group did not exhibit benefits regarding MACCE, death, or MI. SAPT (aspirin or clopidogrel) demonstrated favorable clinical outcomes, and with the development of DES profile, the duration of DAPT maintenance is gradually decreasing. Unlike other studies, we only targeted patients with a second-generation DES, with a thinner strut thickness, and biocompatible or biodegradable polymer coating, to improve arterial healing and decrease the risk of thrombotic adverse events (21); this may reduce the need for prolonged intense platelet inhibition beyond 12 months. Our study was additionally analyzed in an East Asian population, with a lower number of thrombotic events observed than in Westerners (22). In a previous randomized trial including Korean patients who underwent PCI (primarily first-generation DES) exhibiting no adverse clinical events within 12 months, prolonged DAPT (aspirin and clopidogrel) for 12-36 months resulted in a small number of clinical events-including all-cause death, MI, and stroke-without associated benefits (23). Fewer absolute ischemic events observed in East Asians compared with Westerners may therefore reduce the need for prolonged DAPT. We analyzed only aspirin or clopidogrel, which are commonly used in real-world clinical practice. Since the use of DAPT including potent $\mathrm{P}_{2} \mathrm{Y}_{12}$ inhibitors (including ticagrelor and prasugrel) beyond 12 months was excluded, the effect of reducing the ischemic risk of DAPT may be less prominent than in other studies; therefore, in the second-generation era, a large-scale, randomized study to determine the optimal antiplatelet strategy in patients-including Asians and Westerners with stable AMI beyond 12 months-is needed.

Additionally, the SAPT group exhibited favorable clinical outcomes in patients with complex features. Along with improvements in DES profile and adjunct pharmacological treatments, patients with complex lesions could now undergo treatment via PCI in more cases. Although there remains no clear definition of complex features for PCI, several studies and expert consensus commonly include bifurcation with two stents, chronic total occlusion, severely calcified lesions using rotational atherectomy, unprotected left main PCI, implanted long stent, multivessel PCI, and $\geq 3$ stents implanted $(4,24,25)$. Nevertheless, there are limited data regarding the appropriate antiplatelet strategy for complex PCI.

Recently, short DAPT with ticagrelor monotherapy exhibited significantly lower bleeding events, without increasing the ischemic risk in patients with complex PCI or STEMI (24, 26); however, in real-world AMI settings, there is still no concept of complex PCI. Furthermore, chronic total occlusion, or bifurcation with two stents or rotational atherectomy, is rare; therefore, we only included left main PCI, implanted long stent, multivessel PCI, and $\geq 3$ implanted stents, as components of complex features in AMI. Nevertheless, prolonged DAPT did not reduce ischemic risk compared with SAPT. Considering the reduction in the risk of ischemic events, our study results suggest that SAPT could be considered for patients with AMI and complex features who underwent second-generation DES implantation without adverse clinical events for 12 months.

This study has some limitations; first, there is an inherent limitation regarding nonrandomized, observational, registry data, which might have resulted in selection bias. However, we attempted to adjust for the measured confounders of different baseline characteristics through the adjusted analyses, including PS matching. Second, covariates used in PS matching were 
mainly derived from data during the index hospitalization, which did not allow adjustments for the differences in patient characteristics between 12 and 36 months after index hospitalization. Third, since there is no record of bleeding events beyond 12 months, it was difficult to analyze the extent to which bleeding events affect MACCE, including all-cause death. Our study therefore focused on ischemic risk reduction according to antiplatelet strategy beyond 12 months; complex features with a high ischemic burden were analyzed separately. Moreover, among $\mathrm{P}_{2} \mathrm{Y}_{12}$ inhibitors, only clopidogrel was selected for this analysis beyond 12 months due to less bleeding tendency and most used in real world practice. Fourth, the definite reasons for DAPT vs. SAPT selection beyond 12 months is unclear, as it was decided by each clinician considering the balance of ischemic and bleeding risk in individual patients. Fifth, this study was conducted in a dedicated east Asian population and the result of this current study cannot be extrapolated to other ethnicities. Lastly, the KAMIR-NIH registry was designed for 3 years of follow-up; thus, we did not evaluate long-term ischemic risks beyond 3 years.

In conclusion, compared with DAPT with clopidogrel, SAPT using aspirin or clopidogrel alone demonstrated a significantly lower risk of MACCE between 12 and 36 months in patients with AMI treated with second-generation DES who remained uneventful for 12 months. Moreover, a reduction in ischemic risk was also observed for AMI with complex features in the SAPT group. Large-scale, multinational randomized trials are thus required to identify the optimal antiplatelet strategy beyond 12 months in patients with AMI in the second-generation DES era.

\section{DATA AVAILABILITY STATEMENT}

The original contributions presented in the study are included in the article/Supplementary Material, further inquiries can be directed to the corresponding author.

\section{REFERENCES}

1. Valgimigli M, Bueno H, Byrne RA, Collet JP, Costa F, Jeppsson A, et al. 2017 ESC focused update on dual antiplatelet therapy in coronary artery disease developed in collaboration with EACTS. Eur Heart J. (2018) 39:213-60. doi: 10.1093/eurheartj/ehx419

2. Neumann FJ, Sousa-Uva M, Ahlsson A, Alfonso F, Banning AP, Benedetto U, et al. 2018 ESC/EACTS guidelines on myocardial revascularization. Eur Heart J. (2019) 40:87-165. doi: 10.1093/eurheartj/ehy394

3. Suh J, Park DW, Lee JY, Jung IH, Lee SW, Kim YH, et al. The relationship and threshold of stent length with regard to risk of stent thrombosis after drug-eluting stent implantation. JACC Cardiovasc Interv. (2010) 3:383-9. doi: $10.1016 /$ j.jcin.2009.10.033

4. Giustino G, Chieffo A, Palmerini T, Valgimigli M, Feres F, Abizaid A, et al. Efficacy and safety of dual antiplatelet therapy after complex PCI. J Am Coll Cardiol. (2016) 68:1851-64. doi: 10.1016/j.jacc.2016.07.760

5. Yeh RW, Kereiakes DJ, Steg PG, Cutlip DE, Croce KJ, Massaro JM, et al. DAPT Study Investigators. Lesion complexity and outcomes of extended dual antiplatelet therapy after percutaneous coronary intervention. J Am Coll Cardiol. (2017) 70:2213-23. doi: 10.1016/j.jacc.2017.09.011

\section{ETHICS STATEMENT}

The studies involving human participants were reviewed and approved by Chonnam National University Hospital Institutional Review Board, IRB approval number: CNUH-2011172. The patients/participants provided their written informed consent to participate in this study.

\section{AUTHOR CONTRIBUTIONS}

JR and YK: study concept and design, interpretation of results, and preparation of manuscripts. JR, SB, YK, and MJ: data collection. JR, SB, YK, and N-HS: data analysis and statistics. D-KC, J-SK, B-KK, DC, M-KH, MJ, and YJ: validation. YK, D-KC, J-SK, B-KK, D-KC, M-KH, MJ, and YJ: supervision. JR, SB, YK, D-KC, J-SK, B-KK, DC, M-KH, MJ, and YJ: manuscript review and editing. All authors contributed to the article and approved the submitted version.

\section{FUNDING}

This study was supported by a faculty research grant of Yonsei University College of Medicine (6-2020-0161) and research seed money of Internal Medicine in Yongin Severance Hospital.

\section{ACKNOWLEDGMENTS}

The authors thank Medical Illustration and Design, part of the Medical Research Support Services of Yonsei University College of Medicine, for all artistic support related to this work.

\section{SUPPLEMENTARY MATERIAL}

The Supplementary Material for this article can be found online at: https://www.frontiersin.org/articles/10.3389/fcvm. 2021.783344/full\#supplementary-material

6. Ueki Y, Karagiannis A, Zanchin C, Zanchin T, Stortecky S, Koskinas KC, et al. Validation of high-risk features for stent-related ischemic events as endorsed by the 2017 DAPT guidelines. JACC Cardiovasc Interv. (2019) 12:820-30. doi: 10.1016/j.jcin.2018.12.005

7. Hahn JY, Song YB, Oh JH, Chun WJ, Park YH, Jang WJ, et al. SMART-CHOICE Investigators. Effect of P2Y12 inhibitor monotherapy vs dual antiplatelet therapy on cardiovascular events in patients undergoing percutaneous coronary intervention: the SMART-CHOICE. Random Clin Trial JAMA. (2019) 321:2428-37. doi: 10.1001/jama.2019.8146

8. Kim BK, Hong SJ, Cho YH, Yun KH, Kim YH, Suh Y, et al. TICO Investigators. Effect of ticagrelor monotherapy vs ticagrelor with aspirin on major bleeding and cardiovascular events in patients with acute coronary syndrome: the TICO randomized clinical trial. JAMA. (2020) 323:2407-16. doi: 10.1001/jama.2020.7580

9. Jernberg T, Hasvold P, Henriksson M, Hjelm H, Thuresson M, Janzon M. Cardiovascular risk in post-myocardial infarction patients: nationwide real world data demonstrate the importance of a long-term perspective. Eur Heart J. (2015) 36:1163-70. doi: 10.1093/eurheartj/ehu505

10. Rapsomaniki E, Thuresson M, Yang E, Blin P, Hunt P, Chung SC, et al. Using big data from health records from four countries to evaluate chronic disease 
outcomes: a study in 114364 survivors of myocardial infarction. Eur Heart J Qual Care Clin Outcomes. (2016) 2:172-83. doi: 10.1093/ehjqcco/qcw004

11. Kim JH, Chae SC, Oh DJ, Kim HS, Kim YJ, Ahn Y, et al. Multicenter cohort study of acute myocardial infarction in korea-interim analysis of the Korea acute myocardial infarction registry-national institutes of health registry. Circ J. (2016) 80:1427-36. doi: 10.1253/circj.CJ-16-0061

12. Steg PG, James SK, Atar D, Badano LP, Blömstrom-Lundqvist C, Borger MA, et al. ESC guidelines for the management of acute myocardial infarction in patients presenting with ST-segment elevation. Eur Heart J. (2012) 33:2569619. doi: 10.1093/eurheartj/ehs215

13. O'Gara PT, Kushner FG, Ascheim DD, Casey DE Jr, Chung MK, de Lemos JA, et al. 2013 ACCF/AHA guideline for the management of STelevation myocardial infarction. J Am Coll Cardiol. (2013) 61:e78-e140. doi: 10.1016/j.jacc.2012.11.019

14. Thygesen K, Alpert JS, Jaffe AS, Simoons ML, Chaitman BR, Thygesen K, et al. Third universal definition of myocardial infarction. J Am Coll Cardiol. (2012) 60:1581-98. doi: 10.1016/j.jacc.2012.08.001

15. Cutlip DE, Windecker S, Mehran R, Boam A, Cohen DJ, van Es GA, et al. Academic Research Consortium. Clinical end points in coronary stent trials: a case for standardized definitions. Circulation. (2007) 115:2344-51. doi: 10.1161/CIRCULATIONAHA.106.685313

16. Choi KH, Song YB, Lee JM, Lee SY, Park TK, Yang JH, et al. Impact of intravascular ultrasound-guided percutaneous coronary intervention on longterm clinical outcomes in patients undergoing complex procedures. JACC Cardiovasc Interv. (2019) 12:607-20. doi: 10.1016/j.jcin.2019.01.227

17. Bhatt DL, Fox KA, Hacke W, Berger PB, Black HR, Boden WE, et al. Clopidogrel and aspirin versus aspirin alone for the prevention of atherothrombotic events. N Engl J Med. (2006) 354:1706-17. doi: 10.1056/NEJMoa060989

18. Bhatt DL, Flather MD, Hacke W, Berger PB, Black HR, Boden WE, et al. CHARISMA Investigators. Patients with prior myocardial infarction, stroke, or symptomatic peripheral arterial disease in the CHARISMA trial. J Am Coll Cardiol. (2007) 49:1982-8. doi: 10.1016/j.jacc.2007.03.025

19. Mauri L, Kereiakes DJ, Yeh RW, Driscoll-Shempp P, Cutlip DE, Steg PG, et al. Twelve or 30 months of dual antiplatelet therapy after drugeluting stents. N Engl J Med. (2014) 371:2155-66. doi: 10.1056/NEJMoa14 09312

20. Palmerini T, Bruno AG, Gilard M, Morice MC, Valgimigli M, Montalescot $\mathrm{G}$, et al. Risk-benefit profile of longer-than-1-year dual-antiplatelet therapy duration after drug-eluting stent implantation in relation to clinical presentation. Circ Cardiovasc Interv. (2019) 12:e007541. doi: 10.1161/CIRCINTERVENTIONS.118.007541

21. Stefanini GG, Taniwaki M, Windecker S. Coronary stents: novel developments. Heart. (2014) 100:1051-61. doi: 10.1136/heartjnl-2012-303522

22. Kim HS, Kang J, Hwang D, Han JK, Yang HM, Kang HJ, et al. Prasugrelbased de-escalation of dual antiplatelet therapy after percutaneous coronary intervention in patients with acute coronary syndrome (HOST-REDUCE-POLYTECH-ACS). Lancet. (2020) 396:1079-89. doi: 10.1016/S0140-6736(20)31791-8

23. Park SJ, Park DW, Kim YH, Kang SJ, Lee SW, Lee CW, et al. Duration of dual antiplatelet therapy after implantation of drug-eluting stents. $\mathrm{N}$ Engl J Med. (2010) 362:1374-82. doi: 10.1056/NEJMoa1001266

24. Dangas G, Baber U, Sharma S, Giustino G, Mehta S, Cohen DJ, et al. Ticagrelor with or without aspirin after complex PCI. J Am Coll Cardiol. (2020) 75:241424. doi: 10.1016/j.jacc.2020.03.011

25. Wykrzykowska JJ, Kerkmeijer LSM. Complex PCI: time for a universal definition. EuroIntervention. (2020) 16:536-7. doi: 10.4244/EIJV16I7A100

26. Lee SJ, Cho JY, Kim BK, Yun KH, Suh Y, Cho YH, et al. Ticagrelor monotherapy versus ticagrelor with aspirin in patients with ST-segment elevation myocardial infarction. JACC Cardiovasc Interv. (2021) 14:431-40. doi: $10.1016 /$ j.jcin.2020.11.036

Conflict of Interest: The authors declare that the research was conducted in the absence of any commercial or financial relationships that could be construed as a potential conflict of interest.

Publisher's Note: All claims expressed in this article are solely those of the authors and do not necessarily represent those of their affiliated organizations, or those of the publisher, the editors and the reviewers. Any product that may be evaluated in this article, or claim that may be made by its manufacturer, is not guaranteed or endorsed by the publisher.

Copyright (C) 2021 Roh, Bae, Kim, Son, Cho, Kim, Kim, Choi, Hong, Jeong, Jang and the KAMIR-NIH Investigators. This is an open-access article distributed under the terms of the Creative Commons Attribution License (CC BY). The use, distribution or reproduction in other forums is permitted, provided the original author(s) and the copyright owner(s) are credited and that the original publication in this journal is cited, in accordance with accepted academic practice. No use, distribution or reproduction is permitted which does not comply with these terms. 